\title{
Efficacy and Safety of Opinercept Tumor Necrosis Factor Inhibitor Therapy for Drug-Refractory Rheumatoid Arthritis: A Randomized Clinical Trial
}

\author{
Toong-Hua LIANG ${ }^{1}$, Chyou-Shen LEE ${ }^{\circledR}$, Shinn-Shing LEE ${ }^{3}$, Chien-Sheng WU ${ }^{4}(\mathbb{D}$,

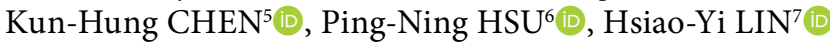 \\ ${ }^{1}$ Department of Internal Medicine, Taipei City Hospital Renai Branch, Taipei, Taiwan \\ ${ }^{2}$ Department of Internal Medicine, Mackay Memorial Hospital, Taipei, Taiwan \\ ${ }^{3}$ Department of Internal Medicine, Cheng Hsin General Hospital, Taipei, Taiwan \\ ${ }^{4}$ Department of Internal Medicine, Far Eastern Memorial Hospital, Taipei, Taiwan \\ ${ }^{5}$ Division of Rheumatology and Immunology, Cathay General Hospital, Taipei, Taiwan \\ ${ }^{6}$ National Taiwan University, Graduate Institute of Immunology, College of Medicine, Taipei, Taiwan \\ ${ }^{7}$ Department of Medicine, Cheng Hsin General Hospital, Taipei, Taiwan
}

\begin{abstract}
Objectives: This study aims to evaluate the efficacy and safety profile of opinercept for rheumatoid arthritis (RA) patients undergoing disease-modifying anti-rheumatic drugs (DMARDs) therapy.

Patients and methods: A total of 98 patients with active RA (17 males, 81 females; mean age $58.6 \pm 12.2$ years; range, 24.3 to 85.3 years) were randomized into opinercept plus DMARDs (OD group) or placebo plus DMARDs (PD group), in a 24-week treatment period. Primary outcome was American College of Rheumatology score (ACR20) at week 24. Other exploratory endpoints included ACR50, ACR70 and disease activity score 28 (DAS28) at week 12 and 24, tender/swollen joint counts, pain, Health Assessment Questionnaire-Disability Index, erythrocyte sedimentation rate, and C-reactive protein level. Incidence of adverse events (AEs), vital signs and physical findings, and laboratory test results were also evaluated.

Results: Patients in OD group showed significantly higher achievement percentage of ACR20 at week 24 than the PD group (76.6\% vs. $30.3 \%$, $\mathrm{p}<0.001$ ). The evaluation of DAS28 was significantly improved in OD patients compared to PD patients at weeks 12 and 24 . Most of the occurred AEs were mild or moderate and considered unrelated to study treatments.

Conclusion: Opinercept concurrent with DMARDs was superior to DMARDs alone in slowing RA progression and ameliorating symptoms, with well-tolerated and acceptable safety profile.

Keywords: Clinical trial, disease-modifying anti-rheumatic drugs, opinercept, rheumatoid arthritis.
\end{abstract}

Rheumatoid arthritis (RA) is a chronic autoimmune disease characterized as joint synovitis. Its manifestations include long-term joint damage, persistent pain, functional impairment, and disability. ${ }^{1,2}$ RA is the most common adult polyarthritis, ${ }^{1,3}$ with estimated global prevalence of $0.35 \%$ and $0.13 \%$ in females and males, respectively. ${ }^{2}$ Prevalence of $\mathrm{RA}$ rises steeply after age 45 and the peak is shown at the age of $70 .{ }^{1}$ Asians have lower prevalence than Europids $(0.16 \%$ vs. $0.45 \%){ }^{2}$ RA incurs substantial burdens of disease and disability, which are positively

Received: March 06, 2019 Accepted: June 24, 2019 Published online: January 08, 2020

Correspondence: Hsiao-Yi Lin, MD. Department of Medicine, Cheng Hsin General Hospital, 11220 Taipei, Taiwan. Tel: +886-3-571-5131 e-mail: doc1116d@gmail.com

Liang TH, Lee CS, Lee SS, Wu CS, Chen KH, Hsu PN, et al. Efficacy and Safety of Opinercept Tumor Necrosis Factor Inhibitor Therapy for Drug-Refractory Rheumatoid Arthritis: A Randomized Clinical Trial. Arch Rheumatol 2020;35(2):170-179. 
associated with population growth and aging. ${ }^{2}$ RA treatment is an important healthcare issue in the world, particularly in Asia, one of the world's fastest-aging regions. ${ }^{4}$

The etiology of RA involves genetic factors, infectious agents, and altered immune responses. ${ }^{5}$ RA pathogenesis begins with an aberrant immune response that triggers inflammation of the synovial lining, followed by increased granulation that causes synovial thickening, nodule formation and vasculitis. As RA progresses, inflamed cells release enzymes that erosively destroy bone and cartilage, causing disability and joint stiffness. ${ }^{5}$ The immune response activates infiltrating T-cells to mobilize early/intermediate inflammatory mediators such as tumor necrosis factor-alpha (TNF- $\alpha$ ), interleukins, and various growth factors. ${ }^{6}$ Meanwhile, antigen-presenting cells such as synovial macrophages and dendritic cells stimulate B-lymphocytes to produce immunoglobins, rheumatoid factors, and complement-complex components. ${ }^{7,8}$ Although pharmacotherapies targeting immune mediators have been developed, traditional supportive treatments like lifestyle modification, physiotherapy, analgesia, and conventional disease-modifying antirheumatic drugs (DMARDs) remain important therapeutic interventions. ${ }^{3,9,10}$ The most widely used DMARDs are immunosuppressants such as methotrexate and leflunomide, and immunomodulators including hydroxychloroquine and sulfasalazine. ${ }^{9,10}$

Besides DMARDs, biological agents including anti-TNF monoclonal antibodies (infliximab) and the TNF inhibitors (etanercept) are also used for RA treatment. ${ }^{3}$ Etanercept is a soluble recombinant TNF-receptor p75 fusion protein (TNFR:Fc) that sequesters extracellular TNF- $\alpha$ to abate signal transduction, ${ }^{11,12}$ which has been approved for treating $\mathrm{RA}$ in the United States, Europe, and Taiwan. Although opinercept is structurally similar to etanercept except for two amino acid differences in the immunoglobulin-G1 heavy chain, ${ }^{13}$ its benefit for RA treatment is still lacking. In the previous phase I/II trials, opinercept has been demonstrated to have acceptable safety and tolerability and improved clinical responses in patients with $\mathrm{RA},{ }^{14,15}$ warranting a corroborative phase III study. Thus, in this study, we aimed to evaluate the efficacy and safety profile of opinercept for RA patients undergoing DMARDs therapy.

\section{PATIENTS AND METHODS}

Seven hospitals conducted this prospective, double-blind, placebo-controlled, randomized parallel group study between September $30^{\text {th }}$ 2013 and December $16^{\text {th }}$ 2015. A total of 98 patients with active RA (17 males, 81 females; mean age $58.6 \pm 12.2$ years; range, 24.3 to 85.3 years) were randomized into two groups to receive opinercept concurrent with DMARDs (OD group) or placebo with DMARDs (PD group) to evaluate the efficacy and safety profile of opinercept in RA treatment. This trial complied with the ethical principles established by the $18^{\text {th }}$ World Medical Assembly (Helsinki, 2008) and applicable amendments, and the International Conference on Harmonisation Good Practice guideline. The study protocol, which also complied with local regulations and guidelines (IRB number: 201304040B, 201303016MSA, 13CT018A, 102028-F, TCH-IRB-1020425, (374)102B-21, CGH-CS102006), was approved by the Taipei Veterans General Hospital, Nation Taiwan University Hospital, Mackay Memorial Hospital, Far Eastern Memorial Hospital, Taipei City Hospital, Cheng Hsin General Hospital, and Cathay General Hospital Ethics Committee. A written informed consent was obtained from each patient or his/her legal representative.

Participants were screened for eligibility within $14 \pm 7$ days before randomization. Besides granting consent and being competent to comply with study procedures, other inclusion criteria were: (i) age $\geq 20$ years with RA functional class I-III by American College of Rheumatology (ACR) criteria for $\geq 6$ months; (ii) currently active disease with at least six tender joints and swollen joints; (iii) erythrocyte sedimentation rate (ESR) $\geq 28 \mathrm{~mm} /$ hour and/or C-reactive protein (CRP) $\geq 10 \mathrm{mg} / \mathrm{L}$; (iv) $\geq 8$ weeks of prior treatment with stable doses of methotrexate, hydroxychloroquine, sulfasalazine, leflunomide, or azathioprine preenrollment.

This study excluded patients (i) with active autoimmune diseases besides RA requiring immunosuppression, documented fibromyalgia, or another joint inflammation disease; (ii) known 
or suspected to have pulmonary tuberculosis or another infectious disease, seropositive for human immunodeficiency virus, or hepatitis viruses $\mathrm{B}$ or $\mathrm{C}$; (iii) adjudged to show persistent signs of immunosuppression; (iv) with a medical history deemed to confer unacceptable risk of significant adverse events (AEs); ( $v$ ) with known hypersensitivity to etanercept or opinercept or their constituents; (vi) unsuccessfully treated with TNF inhibitors; (vii) with serum alanine/aspartate aminotransferase >three times of the upper reference limit, creatinine $>2 \mathrm{mg} / \mathrm{dL}$, leukocytes $<3,000 / \mathrm{mm}^{3}$, platelets $<100,000 / \mathrm{mm}^{3}$, hemoglobin $<8.5 \mathrm{~g} / \mathrm{dL}$; (viii) immunized with an attenuated live vaccine $\leq 3$ months or Bacillus Calmette-Guérin $\leq 12$ months before enrollment; (ix) of childbearing potential and lactating, with a positive screening pregnancy test, or refusing to use reliable contraception during the study; (x) receiving any other investigational agent within 28 days or five half-lives (whichever longer) before commencing study treatment; (xi) with a history of substance addiction or misuse; (xii) who had already participated in an opinercept trial.

At week zero, investigators numbered consecutive enrollees whose eligibility was reconfirmed, and gave them study medication from the pack carrying their corresponding treatment code, which contained either $25 \mathrm{mg}$ opinercept or a physically indistinguishable placebo solution. The Contract Research Organization generated the treatment codes using a randomization table and gave the manufacturer the encryption list, but masked decryption keys from investigators in sealed opaque envelopes (opened only in an emergency), thereby randomizing subjects blindly $2: 1$ to receive opinercept versus placebo. Subsequent visits for study evaluations were scheduled every four weeks during the 24-week treatment period, with follow-up at week 26.

Study subjects took permitted DMARDs as methotrexate 7.5-25.0 mg/week; hydroxychloroquine 200-400 $\mathrm{mg} / \mathrm{day}$ $(\leq 6.5 \mathrm{mg} / \mathrm{kg} /$ day $) ;$ sulfasalazine $\leq 3 \mathrm{~g} /$ day; azathioprine $1.5-3.0 \mathrm{mg} / \mathrm{kg} / \mathrm{day} ;$ and/or leflunomide $10-20 \mathrm{mg} /$ day at stable doses and twice of $1 \mathrm{~mL}$ vials of their study medication weekly. Patients (or caregivers) were instructed to inject study drugs either on the same day or three/four days apart with further supervised administration and training provided as necessary.

Investigators treated coexistent diseases as usual but minimized concomitant medications to avoid potential confounding.

Permitted medications besides on-study DMARDs included: stable prednisolone $\leq 10 \mathrm{mg} /$ day or equivalent since $\geq 4$ weeks before commencing study treatments, including intermittent methylprednisolone; however, intravenous methylprednisolone was suspended 72 hours before assessing joints. Oral non-steroidal anti-inflammatory drugs (NSAIDs) dosed stably as labelled since $\geq 4$ weeks before starting study treatments; low-dose narcotics/analgesics was suspended 24 hours before assessing joints.

Medications prohibited before/during the study included: other anti-arthritis medications from four weeks before study treatment began, adalimumab within two months before starting study treatment, anti-CD20 monoclonal antibody within four months before starting study treatment, attenuated live vaccine, injected NSAIDs, and other investigational products.

Participants would be withdrawn from the project whenever the following criteria were reached, including loss of follow-up, withdrawing consent, serious infection or sepsis, less than 20\% of improvement according to ACR criteria (ACR20) by 12 weeks (enabling opinercept non-responders to withdraw or patients receiving placebo to transfer to an opinercept extension study), death, pregnancy, or the necessary situation determined by the investigator to change anti-rheumatic therapy or prescribe prohibited medications in case that further participation detriments the patient's well-being.

Efficacy assessments were conducted based on rates of improvement by 20\%, 50\% and $70 \%$ according to ACR criteria (ACR20/50/70), and changes from baseline in: disease activity score-28 (DAS28), ACR 66/68 count for tender and swollen joints, pain visual analog scale (VAS), patient and physician global assessments, Health Assessment Questionnaire-Disability Index (HAQ-DI), and levels of ESR and CRP. The primary efficacy endpoint was ACR20 response at week 24. Exploratory endpoints included ACR20 at week 12 and ACR50 and ACR70 at 
weeks 12 and 24; and change from baseline to weeks 12 and 24 in DAS28, and the other efficacy metrics.

Safety endpoints included incidence of AEs (Medical Dictionary for Regulatory Activities Version 18.1, International Council for Harmonisation of Technical Requirements for Pharmaceuticals for Human Use, Geneva, Switzerland), vital signs and physical findings, hematology, serum biochemistry, urinalysis, antiopinercept antibody levels, and Mycobacterium tuberculosis test results.

\section{Statistical analysis}

Based on trials of etanercept, ARC20 rates of approximately $60 \%$ in patients receiving opinercept and $27 \%$ in controls were anticipated, ${ }^{16,17}$ in which 50 patients of OD group and 25 controls would be required to detect significant superiority of opinercept versus placebo with $90 \%$ power at a one-sided significance level of 0.025 . Presuming $20 \%$ of discontinued rate, we randomized 90 patients with the ratio of 2:1 to OD and PD group, and ultimately achieved 75 evaluable patients. The intention-to-treat population was defined as all randomized patients who received $\geq 1$ dose of study medication, regardless of compliance to the study protocol.

Statistical analysis was conducted using SAS Version 9.4 for Windows (SAS Institute, Cary, North Carolina, USA) and Visual FoxPro Version 9.0 (Microsoft, Redmond, Washington, USA). Continuous variables with normal distribution were summarized as means/medians, standard deviations, minimum and maximum, and compared using t-tests or Wilcoxon rank sum tests. Median and range were given for non-normally distributed variables. Categorical variables were summarized as totals, frequencies and proportions, compared by chi-square test and Fisher's exact test, as appropriate. Two-tailed $p$ values of less than 0.05 were considered as statistically significant.

Patients without ACR response observations at study evaluation visits were considered non-responders. Rather than replacing other

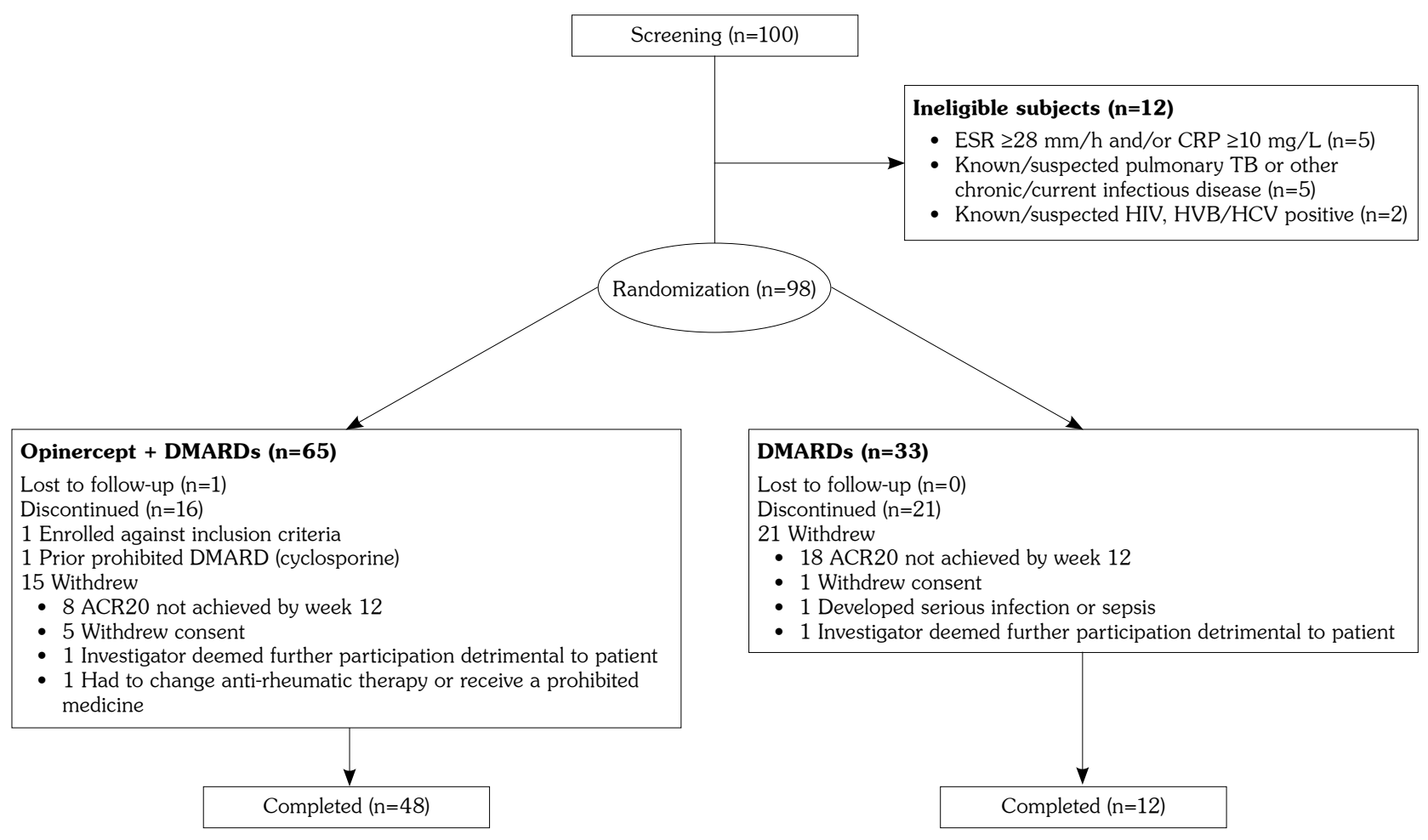

Figure 1. CONSORT (CONsolidated Standards of Reporting Trials) participant flow diagram. Number on left stands for patient numbers at stage or condition.

ESR: Erythrocyte sedimentation rate; CRP: C-reactive protein; TB: Tuberculosis; HIV: Human immunodeficiency virus; HBV/HCV: Hepatitis B/C virus; DMARD: Disease-modifying anti-rheumatic drug; ACR20: 20\% improvement by American College of Rheumatology criteria. 


\begin{tabular}{|c|c|c|c|c|c|c|c|c|c|c|}
\hline \multirow[b]{2}{*}{$\begin{array}{l}\text { Intention-to-treat: efficacy } \\
\text { (randomized patients receiving } \\
\geq 1 \text { double-blind treatment dose)* }\end{array}$} & \multicolumn{5}{|c|}{ Opinercept + DMARDs } & \multicolumn{5}{|c|}{ Placebo + DMARDs } \\
\hline & $\mathrm{n}$ & $\%$ & Mean \pm SD & Median & Range & $\mathrm{n}$ & $\%$ & Mean \pm SD & Median & Range \\
\hline \multicolumn{11}{|l|}{ Ethnic group } \\
\hline Taiwanese/Chinese & 63 & 98.4 & & & & 32 & 97.0 & & & \\
\hline Indigenous Taiwanese & 1 & 1.6 & & & & 0 & 0.0 & & & \\
\hline Vietnamese & 0 & 0.0 & & & & 1 & 3.0 & & & \\
\hline \multicolumn{11}{|l|}{ Sex } \\
\hline Female & 55 & 85.9 & & & & 25 & 75.8 & & & \\
\hline Age (year) & & & $59.0 \pm 11.5$ & 57.9 & $24.3-85.3$ & & & $57.4 \pm 13.4$ & 60.1 & $34.3-83.1$ \\
\hline Duration of rheumatoid arthritis (year) & & & $5.7 \pm 5.5$ & 3.1 & $0.5-21.1$ & & & $7.7 \pm 7.4$ & 5.0 & $0.6-27.2$ \\
\hline \multicolumn{11}{|l|}{ Global functional status } \\
\hline Class I & 23 & 35.9 & & & & 11 & 33.3 & & & \\
\hline Class II & 16 & 25.0 & & & & 10 & 30.3 & & & \\
\hline Class III & 25 & 39.1 & & & & 12 & 36.4 & & & \\
\hline 0 & 0 & 0.0 & & & & 0 & 0.0 & & & \\
\hline $1-3$ & 46 & 71.9 & & & & 25 & 75.8 & & & \\
\hline$\geq 4$ & 18 & 28.1 & & & & 8 & 24.2 & & & \\
\hline \multicolumn{11}{|c|}{ Number and types of DMARDs ever received } \\
\hline Hydroxychloroquine & 59 & 92.2 & & & & 32 & 97.0 & & & \\
\hline Methotrexate & 56 & 87.5 & & & & 26 & 78.8 & & & \\
\hline Leflunomide & 27 & 42.2 & & & & 14 & 42.4 & & & \\
\hline Sulfasalazine & 3 & 4.7 & & & & 2 & 6.1 & & & \\
\hline Azathioprine & 1 & 1.6 & & & & 21 & 6.1 & & & \\
\hline Cyclosporine & 2 & 3.1 & & & & 1 & 3.0 & & & \\
\hline D-penicillamine & 0 & 0.0 & & & & 1 & 3.0 & & & \\
\hline
\end{tabular}

DMARD: Disease-modifying anti-rheumatic drug; SD: Standard deviation; " Irrespective of protocol compliance; however excluding one patient mistakenly randomized despite not fulfilling entry criteria.

missing efficacy data using the last observation carried forward method, we used multiple imputation (monotone multiple regression), which is more rigorous with less bias. ${ }^{18,19}$ Missing safety data were not replaced.

\section{RESULTS}

Starting from September $30^{\text {th }} 2013$, this study randomized 98 patients (17 males, 81 females; mean age $58.6 \pm 12.2$ years; range, 24.3 to 85.3 years) from participating hospitals to receive permitted treatment with DMARDs plus either opinercept (OD group) or placebo (PD group) (Figure 1). All participants received at least one treatment dose, with in average $83 \%$ of compliance rate in OD group. Sixty patients (10 males, 50 females; mean age $56.6 \pm 11.3$ years; range, 24.3 to 78.4 years) completed the study. The most common reason for discontinuation in the earlier stage was not achieving ACR20 by week 12 (38 patients).

There was no notable between-group difference in baseline characteristics (Table 1).
Almost all subjects were Taiwanese/Chinese, predominantly female and middle-aged. Patients were roughly evenly distributed to three classes of global functional status. The most frequently prescribed DMARDs for patients during study were hydroxychloroquine (71.4\%), methotrexate (58.2\%), sulfasalazine (25.5\%), and leflunomide (20.4\%).

Patients in OD group received significantly higher proportions of ACR20 responses than those in PD group at all time points $(p<0.001)$ (Figure 2). Likewise, patients in OD group also had relatively higher proportions of ACR50 responses throughout the study compared to PD group at weeks 12 and $24(p \leq 0.01)$. Although lower, ACR70 response rates showed a similar pattern as shown in ACR20 and ACR50, with statistically significant differences at weeks 12 and $24(p<0.05)$.

Patients in OD group showed significantly lower scores in DAS28 from week four to week 12 compared with patients in PD group $(p<0.05)$, indicating obvious improvement in disease severity (Table 2). Patients in the OD group consistently 


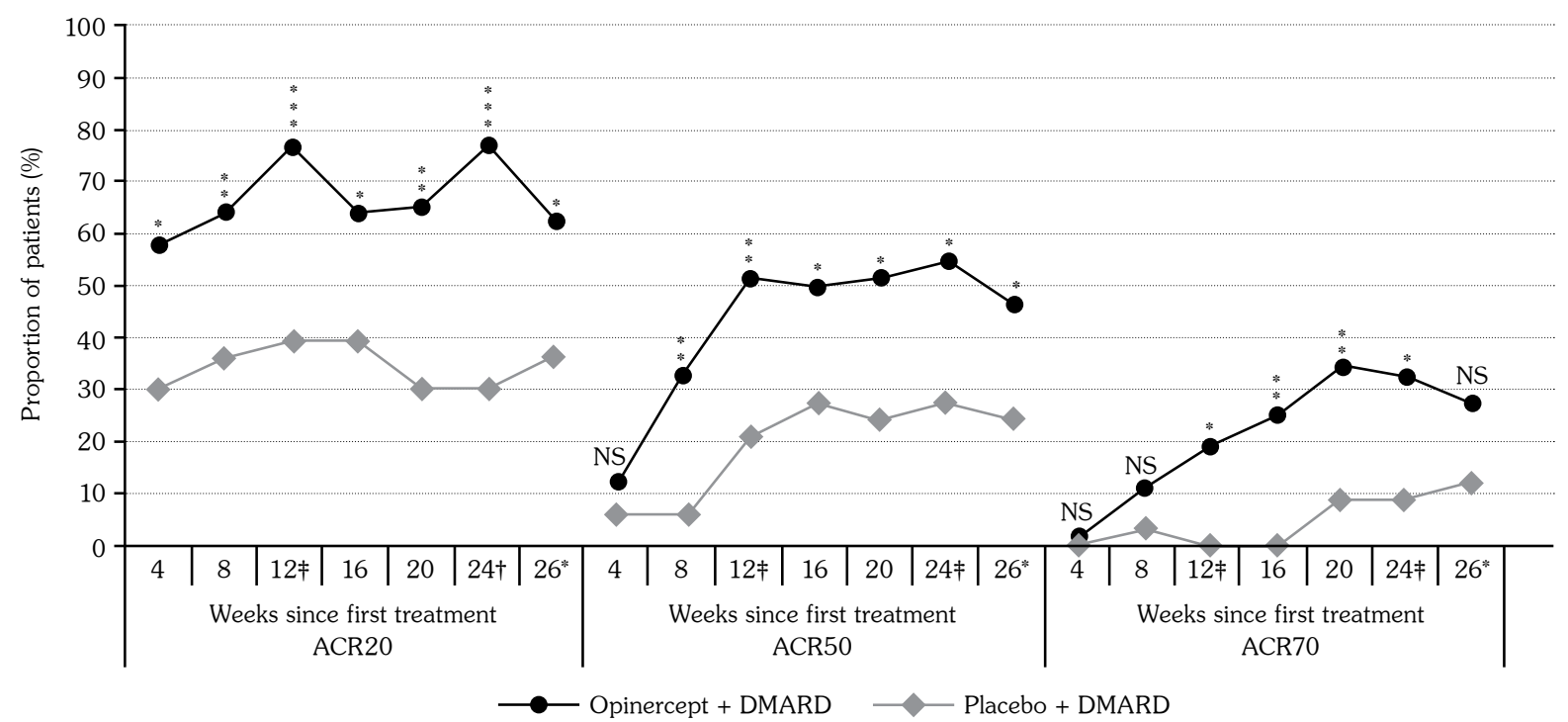

Figure 2. American College of Rheumatology (ACR) response rates. Response rates in opinercept plus disease-modifying anti-rheumatic drugs group were significantly better than placebo plus disease-modifying antirheumatic drugs group in ACR20, ACR50 and ACR70 assessments.

DMARD: Disease-modifying anti-rheumatic drug; ACR20/50/70: 20\%, 50\%, or 70\% improvement by American College of Rheumatology Criteria; NS: Not significant; † Primary endpoint (includes early termination); $\neq$ Exploratory endpoint; ${ }^{*}$ Post-treatment follow-up; ${ }^{*}$ p $<0.05$; *** $\mathrm{p}<0.01 ;{ }^{* * * *} \mathrm{p}<0.001$.

reported less pain and fewer tender/swollen joints at subsequent time points (all $\mathrm{p}<0.001$ ), with significantly greater improvements than patients in PD group at week 12 and $24(p<0.05)$.

Changes in physician and patient global assessment and HAQ-DI scores were significantly greater in patients in OD group than those in PD group at week $12(\mathrm{p}<0.05)$, but not at week 24 (Table 2).

Level of ESR and CRP were significantly reduced from baseline throughout the study in OD group ( $p \leq 0.001)$. When compared with PD group, changes of ESR and CRP were significantly higher in OD group at week $12(\mathrm{p}<0.05)$ but not at week 24 (Table 2).

Each treatment group showed a similar incidence of AEs (Table 3). More than 95\% of AEs in either group were mild or moderate, and most were not attributed to the study treatments. Three AEs which were highly likely related to opinercept treatment were delayed wound healing, urinary tract infection (UTI), and a herpes simplex outbreak. The only severe $\mathrm{AE}$ in the placebo arm was tuberculosis of the knee-joint, possibly treatment-related. The most common class of $\mathrm{AE}$ in either treatment group was infections and infestations, among which UTI and upper respiratory tract infection were the only specific AEs that affected more than $5 \%$ of patients in the placebo arm.

No patients died. In OD group, four patients had five serious adverse events (SAEs), but only one with a fracture due to a fall was rated as severe, which was not drug-related. One moderate SAE of fever due to UTI was possibly drug-related. One mild SAE was fever due to suspected pneumonia, which is unlikely to be drug-related. The other two mild SAEs were poor wound healing and UTI, which were highly-probably drug-related. On the other hand, there were two moderate SAEs in PD group, which were intermittent shortness of breath and multiple closed rib fractures, both were considered unrelated to study drugs.

Results of hematology, biochemistry, urinalysis, vital signs and physical examinations in both groups were generally stable and within their normal ranges, and changes from baseline were mostly slight without clinical significance. Antibody against opinercept was not detected. 
Table 2. Changes in DAS28, tender/swollen joints, VAS, physician and patient global assessments, HAQ-DI, ESR, and CRP

\begin{tabular}{|c|c|c|c|c|c|c|c|c|}
\hline \multirow{2}{*}{$\begin{array}{l}\text { ITT: Efficacy (randomized patients } \\
\text { taking } \geq 1 \text { double-blind treatment } \\
\text { dose) })^{*}\end{array}$} & \multicolumn{3}{|c|}{ Opinercept + DMARDs $(n=64)$} & \multicolumn{3}{|c|}{ Placebo + DMARDs ( $\mathrm{n}=33)$} & \multicolumn{2}{|c|}{$\begin{array}{l}\text { P Value } \neq: \text { Opinercept vs } \\
\text { Placebo }\end{array}$} \\
\hline & Mean \pm SD & Mean $\pm \mathrm{SD}^{\wedge}$ & $P$ value $\dagger$ & Mean \pm SD & Mean $\pm \mathrm{SD}^{\Delta}$ & $P$ value $\dagger$ & Mean & Change \\
\hline \multicolumn{9}{|l|}{$\begin{array}{l}\text { Change in DAS } 28 \text { by visits at } \\
\text { week: }\end{array}$} \\
\hline Baseline & $6.4 \pm 1.2$ & NA & NA & $5.9 \pm 1.0$ & NA & NA & 0.032 & NA \\
\hline $12^{\pi}$ & $3.7 \pm 1.5$ & $-2.7 \pm 1.6$ & $<0.001$ & $4.8 \pm 1.5$ & $-1.0 \pm 1.3$ & $<0.001$ & 0.001 & $<0.001$ \\
\hline 24/early termination ${ }^{\top}$ & $3.2 \pm 1.1$ & $-3.2 \pm 1.6$ & $<0.001$ & $3.5 \pm 1.0$ & $-2.4 \pm 1.3$ & $<0.001$ & 0.23 & 0.013 \\
\hline \multicolumn{9}{|l|}{$\begin{array}{l}\text { Change in number of tender joints } \\
\text { by visits at week: }\end{array}$} \\
\hline Baseline & $19.0 \pm 12.1$ & NA & NA & $14.5 \pm 9.2$ & NA & NA & 0.067 & NA \\
\hline $12^{\text {वा}}$ & $5.9 \pm 9.2$ & $-13.1 \pm 11.0$ & $<0.001$ & $9.2 \pm 12.1$ & $-5.4 \pm 12.4$ & 0.018 & 0.14 & 0.002 \\
\hline 24/early termination ${ }^{\top}$ & $2.6 \pm 3.8$ & $-16.3 \pm 11.5$ & $<0.001$ & $5.9 \pm 7.7$ & $-8.6 \pm 9.2$ & $<0.001$ & 0.026 & 0.001 \\
\hline \multicolumn{9}{|l|}{$\begin{array}{l}\text { Change in number of swollen joints } \\
\text { by visits at week: }\end{array}$} \\
\hline Baseline & $14.1 \pm 8.5$ & NA & NA & $10.6 \pm 5.5$ & NA & NA & 0.015 & NA \\
\hline $12^{\pi}$ & $3.2 \pm 4.8$ & $-10.9 \pm 8.2$ & $<0.001$ & $6.5 \pm 8.2$ & $-4.0 \pm 7.3$ & 0.003 & 0.037 & $<0.001$ \\
\hline 24/early termination ${ }^{\top}$ & $1.9 \pm 2.8$ & $-12.2 \pm 8.1$ & $<0.001$ & $3.6 \pm 3.2$ & $-7.0 \pm 6.5$ & $<0.001$ & 0.008 & 0.002 \\
\hline \multicolumn{9}{|l|}{$\begin{array}{l}\text { Change in pain VAS by visits at } \\
\text { week: }\end{array}$} \\
\hline Baseline & $6.8 \pm 1.9$ & NA & NA & $6.0 \pm 1.9$ & NA & NA & 0.066 & NA \\
\hline $12^{\pi}$ & $3.6 \pm 2.3$ & $-3.2 \pm 2.6$ & $<0.001$ & $5.2 \pm 2.3$ & $-0.9 \pm 1.9$ & 0.011 & 0.002 & $<0.001$ \\
\hline 24/early termination ${ }^{\top}$ & $2.7 \pm 1.8$ & $-4.1 \pm 2.3$ & $<0.001$ & $2.5 \pm 1.8$ & $-3.5 \pm 2.5$ & $<0.001$ & 0.64 & 0.25 \\
\hline \multicolumn{9}{|l|}{$\begin{array}{l}\text { Change in physician global } \\
\text { assessments by visits at week: }\end{array}$} \\
\hline Baseline & $8.0 \pm 1.4$ & NA & NA & $7.9 \pm 1.1$ & NA & NA & 0.85 & NA \\
\hline $12^{\text {वा }}$ & $3.8 \pm 2.6$ & $-4.2 \pm 2.7$ & $<0.001$ & $5.5 \pm 2.4$ & $-2.4 \pm 2.4$ & $<0.001$ & 0.003 & 0.003 \\
\hline 24/early termination ${ }^{\top}$ & $3.6 \pm 2.7$ & $-4.4 \pm 3.0$ & $<0.001$ & $3.8 \pm 2.2$ & $-4.2 \pm 1.9$ & $<0.001$ & 0.84 & 0.75 \\
\hline \multicolumn{9}{|l|}{$\begin{array}{l}\text { Change in patient global } \\
\text { assessments by visits at week: }\end{array}$} \\
\hline Baseline & $6.7 \pm 2.2$ & NA & NA & $6.1 \pm 2.0$ & NA & NA & 0.20 & NA \\
\hline $12^{\pi}$ & $3.6 \pm 2.2$ & $-3.1 \pm 2.6$ & $<0.001$ & $5.0 \pm 2.2$ & $-1.1 \pm 1.9$ & 0.002 & 0.003 & $<0.001$ \\
\hline 24/early termination ${ }^{\top}$ & $2.9 \pm 1.8$ & $-3.8 \pm 2.6$ & $<0.001$ & $1.6 \pm 1.2$ & $-4.5 \pm 2.1$ & $<0.001$ & $<0.001$ & 0.15 \\
\hline \multicolumn{9}{|l|}{$\begin{array}{l}\text { Change in HAQ-DI by visits at } \\
\text { week: }\end{array}$} \\
\hline Baseline & $1.6 \pm 0.7$ & NA & NA & $1.2 \pm 0.8$ & NA & NA & 0.029 & NA \\
\hline $12^{\pi}$ & $0.95 \pm 0.66$ & $-0.61 \pm 0.55$ & $<0.001$ & $1.06 \pm 0.71$ & $-0.15 \pm 0.47$ & 0.067 & 0.44 & $<0.001$ \\
\hline 24/early termination ${ }^{\top}$ & $0.82 \pm 0.64$ & $-0.75 \pm 0.60$ & $<0.001$ & $0.66 \pm 0.52$ & $-0.55 \pm 0.45$ & $<0.001$ & 0.23 & 0.11 \\
\hline \multicolumn{9}{|l|}{ Change in ESR by visits at week: } \\
\hline Baseline & $47.1 \pm 26.3$ & NA & NA & $42.6 \pm 27.2$ & NA & NA & 0.43 & NA \\
\hline $12^{\text {या }}$ & $27.3 \pm 22.9$ & $-19.8 \pm 20.7$ & $<0.001$ & $40.3 \pm 27.6$ & $-2.3 \pm 24.3$ & 0.60 & 0.015 & $<0.001$ \\
\hline 24/early termination ${ }^{\mathrm{T}}$ & $26.2 \pm 20.7$ & $-20.9 \pm 20.3$ & $<0.001$ & $18.3 \pm 11.1$ & $-24.3 \pm 25.9$ & $<0.001$ & 0.016 & 0.48 \\
\hline \multicolumn{9}{|l|}{ Change in CRP by visits at week: } \\
\hline Baseline & $2.7 \pm 3.0$ & NA & NA & $1.8 \pm 2.2$ & NA & NA & 0.12 & NA \\
\hline $12^{\pi}$ & $1.1 \pm 1.7$ & $-1.6 \pm 2.5$ & $<0.001$ & $1.7 \pm 1.6$ & $-0.1 \pm 2.3$ & 0.81 & 0.066 & 0.005 \\
\hline 24/early termination ${ }^{\top}$ & $1.0 \pm 1.6$ & $-1.7 \pm 2.6$ & $<0.001$ & $0.6 \pm 0.6$ & $-1.2 \pm 2.2$ & 0.004 & 0.11 & 0.37 \\
\hline
\end{tabular}

DAS28: Disease activity score-28; VAS: Visual analog scale; HAQ-DI: Health Assessment Questionnaire-Disability Index; ESR: Erythrocyte sedimentation rate; CRP: C-reactive protein; ITT: Intention-to-treat; $\Delta$ : Change; NA: Not applicable; ${ }^{*}$ Irrespective of protocol compliance; † Paired t-test; † T-test; 91 Exploratory efficacy endpoint. 
Table 3. Adverse events

\begin{tabular}{|c|c|c|c|c|c|c|c|c|}
\hline \multirow{3}{*}{$\begin{array}{l}\text { Intention-to-treat safety population }(\mathrm{n}=98)^{*} \\
\text { All data show: No. }(\%) \\
\text { Adverse event category }\end{array}$} & \multicolumn{4}{|c|}{ Opinercept + DMARDs $(n=65) \dagger$} & \multicolumn{4}{|c|}{ Placebo + DMARDs $(n=33)$} \\
\hline & \multicolumn{2}{|c|}{$\mathrm{AE}$} & \multicolumn{2}{|c|}{ SAE } & \multicolumn{2}{|c|}{$\mathrm{AE}$} & \multicolumn{2}{|c|}{ SAE } \\
\hline & $\mathrm{n}$ & $\%$ & $\mathrm{n}$ & $\%$ & $\mathrm{n}$ & $\%$ & $\mathrm{n}$ & $\%$ \\
\hline Patients with adverse event(s) & 39 & 60.0 & 4 & 6.2 & 19 & 57.6 & 2 & 6.1 \\
\hline $\begin{array}{l}\text { Number and severity of adverse events } \\
\text { Total } \\
\text { Mild } \\
\text { Moderate } \\
\text { Severe }\end{array}$ & $\begin{array}{l}70 \\
61 \\
8 \\
1\end{array}$ & $\begin{array}{c}100 \\
87.1 \\
11.4 \\
1.4\end{array}$ & $\begin{array}{l}5 \\
3 \\
1 \\
1\end{array}$ & $\begin{array}{l}100 \\
60.0 \\
20.0 \\
20.0\end{array}$ & $\begin{array}{c}26 \\
21 \\
4 \\
1\end{array}$ & $\begin{array}{l}100 \\
80.8 \\
15.4 \\
3.8\end{array}$ & $\begin{array}{l}2 \\
0 \\
2 \\
0\end{array}$ & $\begin{array}{l}100 \\
0.0 \\
100 \\
0.0\end{array}$ \\
\hline $\begin{array}{l}\text { Assessed relationship of adverse events } \\
\text { with opinercept administration } \\
\text { Highly probable } \\
\text { Probable } \\
\text { Possible } \\
\text { Unlikely } \\
\text { Unrelated }\end{array}$ & $\begin{array}{c}3 \\
4 \\
12 \\
9 \\
42\end{array}$ & $\begin{array}{l}4.3 \\
5.7 \\
17.1 \\
12.9 \\
60.0\end{array}$ & $\begin{array}{l}2 \\
0 \\
1 \\
1 \\
1\end{array}$ & $\begin{array}{c}40.0 \\
0.0 \\
20.0 \\
20.0 \\
20.0\end{array}$ & $\begin{array}{c}0 \\
2 \\
8 \\
3 \\
13\end{array}$ & $\begin{array}{c}0.0 \\
7.7 \\
30.8 \\
11.5 \\
50.0\end{array}$ & $\begin{array}{l}0 \\
0 \\
0 \\
0 \\
1\end{array}$ & $\begin{array}{r}0.0 \\
0.0 \\
0.0 \\
0.0 \\
100\end{array}$ \\
\hline $\begin{array}{l}\text { Adverse events affecting }>5 \% \text { of patients } \\
\text { in either treatment arm: MedDRA }{ }^{\circledast} \\
\text { System Organ Class/Preferred Term } \\
\text { Infections \& infestations/ } \\
\text { Upper respiratory tract } \\
\text { Urinary tract infection } \\
\text { Skin \& subcutaneous tissue } \\
\text { Gastrointestinal disorders } \\
\text { Investigations } \neq \\
\text { General \& administration site conditions } \\
\text { Nervous system disorders } \\
\text { Respiratory, thoracic \& mediastinal } \\
\text { Injury, poisoning \& procedural complications }\end{array}$ & $\begin{array}{l}15 \\
2 \\
9 \\
6 \\
6 \\
4 \\
7 \\
3 \\
3 \\
1\end{array}$ & $\begin{array}{c}23.1 \\
3.1 \\
13.8 \\
9.2 \\
9.2 \\
6.2 \\
10.8 \\
4.6 \\
4.6 \\
1.5\end{array}$ & & & $\begin{array}{l}9 \\
3 \\
3 \\
3 \\
1 \\
1 \\
1 \\
3 \\
2 \\
2\end{array}$ & $\begin{array}{c}27.3 \\
9.1 \\
9.1 \\
9.1 \\
3.0 \\
3.0 \\
3.0 \\
9.1 \\
6.1 \\
6.1\end{array}$ & & \\
\hline
\end{tabular}

Most of the patients remained in similar physical condition throughout the study. Regarding the RA symptom, $81.5 \%$ and $77.8 \%$ of patients in OD and PD group, respectively, had abnormal musculoskeletal findings at baseline, and 7.8\% and $8.3 \%$ of patients in OD and PD group, respectively, felt improvements at week 24 .

\section{DISCUSSION}

This study provides new data on TNFR:Fc therapy in Asian patients with RA, which are commensurate with outcomes in other studies of etanercept plus DMARD therapy, including some involving Asian subjects. ${ }^{20-23}$ Opinercept plus DMARDs was superior to DMARDs alone for treating RA in Taiwanese/Chinese patients, with acceptable safety. These results support the evidence-base for local registration.
Opinercept inhibits TNF- $\alpha$ signaling in the same way as etanercept. Since the subjects in this study had generally similar characteristics to those in previous etanercept trials in terms of age, sex, and prior RA treatments, albeit with relatively short duration of $\mathrm{RA},{ }^{17}$ it is valid to compare the findings in this study with those of similar studies with etanercept. The efficacy outcomes of opinercept were very similar to those in a 52-week comparative study of etanercept versus methotrexate in patients with active RA in Japan, in which the response in ACR20, improvements in DAS28, and ratio of tender or swollen joints were $79 \%, 43 \%$ and $74 \%$, respectively. ${ }^{22}$ The improvement of HAQ-DI between OD and PD groups in this study was also comparable with that reported for etanercept plus methotrexate versus methotrexate combined with other DMARDs. ${ }^{23}$ Therefore, clinical outcomes in this study are probably generalizable beyond this study cohort. 
Patients treated with opinercept had significantly higher ACR20, ACR50 and ACR70 rates at all time points (Figure 2). Compared with previous reports, improved ACR responses in OD group versus PD group were evidently earlier, from week four onward. With regards to the rate of withdrawals, patients in PD group had four times higher rate due to failing to achieve ACR20 by week 12 compared with the patients in PD group. Following early peak activity, ACR response rates appeared to plateau from around week 20 , as also occurs in study of etanercept. ${ }^{17}$ Treatment of opinercept plus DMARDs was also more efficacious than DMARDs alone in this study in slowing joint damage and disease progression, with faster treatment responses and relative improvements after 24-weeks' treatment.

Treatment with opinercept plus DMARDs was generally well-tolerated, with no unexpected safety findings as in the treatment with DMARDs alone. Most of the AEs were mild and were considered unrelated to study treatment, with similar patterns of severity and causality in both treatment groups, in common with etanercept. The most frequent AEs were infections and infestations, which might be due to the immunosuppressant activity of opinercept which potentially compromises resistance to infections. More than 5\% of patients in each treatment group had UTIs, and more than 5\% in the PD group had an upper respiratory infection. However, patients in OD group showed slightly lower incidence of UTIs and upper respiratory infection than those in PD group, indicating that adding opinercept neither increased the risk of infections, nor exacerbated them. Although three SAEs in two patients were suspected to be associated with the use of opinercept, these patients were fully recovered. Other safety outcomes were comparable between treatment arms, suggesting no clinically significant risk from adding opinercept to DMARDs.

This study reflects the efficacy and tolerable side effects of the combination therapy of TNFR:Fc and DMARDs in RA patients, which has been a standard regimen as recommended in the international guidelines. ${ }^{24}$ However, even with the multiple imputation for the missing data, due to the higher withdrawal rate in the placebo arm because of the non-achievement of ACR20, the efficacy in treatment group might be underestimated. ${ }^{19}$ This potentially explains why some exploratory endpoints were significant at week 12 but not at week 24 .

In conclusion, administering opinercept concurrently with DMARDs was significantly superior to DMARDs alone in slowing disease progression and ameliorating clinical symptoms in patients with active RA. Combination therapy was well-tolerated, with an acceptable safety profile. These findings support the rationale for adding opinercept to conventional DMARDs for RA treatment, which contributes a more effective way and benefits patients' quality of life.

\section{Acknowledgements}

The authors thank Dr. David Neil (PhD), of Content Ed Net (Taiwan) for professional medical writing services, which were remunerated by Mycenax Biotech Corporation. Ltd., Taiwan.

\section{Declaration of conflicting interests}

The authors declared no conflicts of interest with respect to the authorship and/or publication of this article.

\section{Funding}

Toong-Hua Liang reported receiving personal fees from Mycenax Biotech. Chyou-Shen Lee reported receiving personal fees from Chugai, Tanabe and UCB. Shinn-Shing Lee reported receiving personal fees from AbbVie, Amgen, Astellas, Bristol-Myers Squobb, Chugai, Pfizer, Novartis, Roche and Johnson \& Johnson. ChienSheng Wu reported receiving personal fees from AbbVie, Bristol-Myers Squibb, Chugai, Eisai, Johnson \& Johnson, Tanabe, Novartis, Pfizer, Roche and Mycenax Biotech. Chien-Sheng Wu reported receiving grant support from AbbVie and Mycenax Biotech. Kun-Hung Chen reported receiving personal fees from AbbVie, Bristol-Myers Squobb, Chugai, Pfizer, Novartis, Tanabe, Mycenax Biotech and UCB. Ping-Ning Hsu reported receiving personal fees from AbbVie, Pfizer, Roche, Bristol-Myers Squibb, Novartis, Johnson \& Johnson and Mycenax Biotech. Ping-Ning Hsu reported receiving grant support from AbbVie, Johnson \& Johnson, Pfizer and Mycenax Biotech. Hsiao-Yi Lin reported receiving personal fees from Mycenax Biotech.

\section{REFERENCES}

1. Woolf AD, Pfleger B. Burden of major musculoskeletal conditions. Bull World Health Organ 2003;81:646-56.

2. Cross M, Smith E, Hoy D, Carmona L, Wolfe F, Vos $\mathrm{T}$, et al. The global burden of rheumatoid arthritis: estimates from the global burden of disease 2010 study. Ann Rheum Dis 2014;73:1316-22. 
3. Singh JA, Saag KG, Bridges SL Jr, Akl EA, Bannuru RR, Sullivan MC, et al. 2015 American College of Rheumatology Guideline for the Treatment of Rheumatoid Arthritis. Arthritis Rheumatol 2016;68:1-26.

4. Fu S, Huang N, Chou YJ. Trends in the prevalence of multiple chronic conditions in Taiwan from 2000 to 2010: a population-based study. Prev Chronic Dis 2014;11:E187.

5. Guzov II. Current concepts on the etiology, pathogenesis and treatment of rheumatoid arthritis. Klin Med (Mosk) 1985;63:24-8.

6. Tran CN, Lundy SK, Fox DA. Synovial biology and $\mathrm{T}$ cells in rheumatoid arthritis. Pathophysiology 2005; 12:183-9.

7. Silverman GJ, Carson DA. Roles of B cells in rheumatoid arthritis. Arthritis Res Ther 2003;5:1-6.

8. Browning JL. B cells move to centre stage: novel opportunities for autoimmune disease treatment. Nat Rev Drug Discov 2006;5:564-76.

9. American College of Rheumatology Subcommittee on Rheumatoid Arthritis Guidelines. Guidelines for the management of rheumatoid arthritis: 2002 Update. Arthritis Rheum 2002;46:328-46.

10. Wolfe F, Cush JJ, O'Dell JR, Kavanaugh A, Kremer $\mathrm{JM}$, Lane NE, et al. Consensus recommendations for the assessment and treatment of rheumatoid arthritis. J Rheumatol 2001;28:1423-30.

11. Moreland LW, Baumgartner SW, Schiff MH, Tindall EA, Fleischmann RM, Weaver AL, et al. Treatment of rheumatoid arthritis with a recombinant human tumor necrosis factor receptor (p75)-Fc fusion protein. N Engl J Med 1997;337:141-7.

12. Moreland LW. Soluble tumor necrosis factor receptor (p75) fusion protein (ENBREL) as a therapy for rheumatoid arthritis. Rheum Dis Clin North Am 1998;24:579-91.

13. Moreland LW. Soluble tumor necrosis factor receptor (p75) fusion protein (ENBREL) as a therapy for rheumatoid arthritis. Rheum Dis Clin North Am 1998;24:579-91.

14. Gu N, Yi S, Kim TE, Kim J, Shin SG, Jang IJ, et al. Comparative pharmacokinetics and tolerability of branded etanercept $(25 \mathrm{mg})$ and its biosimilar $(25 \mathrm{mg})$ : a randomized, open-label, single-dose, two-sequence, crossover study in healthy Korean male volunteers. Clin Ther 2011;33:2029-37.

15. Chen DY, Lai NS, Lu LY, Chou HC, Chen YH, Hsieh TY, et al. A multicenter open-label phase I/II study to assess the safety, tolerability, and efficacy of three dose levels of TuNEX in patients with rheumatoid arthritis. J Chin Med Assoc 2011;74:544-51.

16. Genovese MC, McKay JD, Nasonov EL, Mysler EF, da Silva NA, Alecock E, et al. Interleukin-6 receptor inhibition with tocilizumab reduces disease activity in rheumatoid arthritis with inadequate response to disease-modifying antirheumatic drugs: the tocilizumab in combination with traditional diseasemodifying antirheumatic drug therapy study. Arthritis Rheum 2008;58:2968-80.

17. Genovese MC, Bathon JM, Martin RW, Fleischmann RM, Tesser JR, Schiff MH, et al. Etanercept versus methotrexate in patients with early rheumatoid arthritis: two-year radiographic and clinical outcomes. Arthritis Rheum 2002;46:1443-50.

18. Dziura JD, Post LA, Zhao Q, Fu Z, Peduzzi P. Strategies for dealing with missing data in clinical trials: from design to analysis. Yale $\mathrm{J}$ Biol Med 2013;86:343-58.

19. Boers M. Missing data in trials: do we have to keep carrying the last observation forward? Arthritis Rheum 2008;59:2-3.

20. Lethaby A, Lopez-Olivo MA, Maxwell L, Burls A, Tugwell P, Wells GA. Etanercept for the treatment of rheumatoid arthritis. Cochrane Database Syst Rev 2013;5:CD004525.

21. Weinblatt ME, Kremer JM, Bankhurst AD, Bulpitt KJ, Fleischmann RM, Fox RI, et al. A trial of etanercept, a recombinant tumor necrosis factor receptor:Fc fusion protein, in patients with rheumatoid arthritis receiving methotrexate. $\mathrm{N}$ Engl J Med 1999;340:253-9.

22. Takeuchi T, Miyasaka N, Zang C, Alvarez D, Fletcher $\mathrm{T}$, Wajdula $\mathrm{J}$, et al. A phase 3 randomized, doubleblind, multicenter comparative study evaluating the effect of etanercept versus methotrexate on radiographic outcomes, disease activity, and safety in Japanese subjects with active rheumatoid arthritis. Mod Rheumatol 2013;23:623-33.

23. Bae SC, Gun SC, Mok CC, Khandker R, Nab HW, Koenig AS, et al. Improved health outcomes with etanercept versus usual DMARD therapy in an Asian population with established rheumatoid arthritis. BMC Musculoskelet Disord 2013;14:13.

24. Singh JA, Furst DE, Bharat A, Curtis JR, Kavanaugh AF, Kremer JM, et al. 2012 update of the 2008 American College of Rheumatology recommendations for the use of disease-modifying antirheumatic drugs and biologic agents in the treatment of rheumatoid arthritis. Arthritis Care Res (Hoboken) 2012;64:625-39. 